\title{
Scanning Microwave Microscopy Studies of Metal-Insulator Transition at Ferroelastic Domain Walls in $\mathrm{VO}_{2}$
}

\author{
A. Tselev, ${ }^{*}$ E. Stelcov, ${ }^{* *}$ K. Jones, ${ }^{* * *}$ R. Proksch, ${ }^{* * *}$ A. Kolmakov, ${ }^{* *}$ S. V. Kalinin* \\ * Oak Ridge National Laboratory, Oak Ridge, TN 37831 \\ ** Physics Department, Southern Illinois State University, Carbondale, IL 62901 \\ *** Asylum Research, Santa Barbara, CA 93117
}

Near-field scanning microwave microscopy (SMM) has established as a useful tool in studies of materials with its unique capability to couple directly to the material ground state through electrodynamical (dielectric function and electric conductivity) properties $[1,2]$. In this talk, after briefly discussing general principles of SMM, we will demonstrate application of this technique to few material systems, in particular to metal-insulator transition in vanadium dioxide nanoplatelets.

The results have been obtained with an SMM system synergistically incorporated into an Asylum Research MFP-3D atomic force microscope platform. As illustrated in Fig. 1, the probe of the system is a solid wire AFM-type cantilever tip on a specially designed holder, which is connected to a coaxial cable (transmission line) through an impedance-matching network and then to a vector network analyzer (VNA). Amplitude and phase of the microwave signal reflected from the tip are measured with the VNA while scanning over the sample surface in contact AFM mode. AFM topography image and the SMM image are acquired simultaneously. The complex value of the reflection coefficient is determined by the capacitance of the system tip-substrate-outer conductor of the coaxial cable, which in turn is defined by the electric properties of the material under the tip apex. Imaging resolution of the system in high-frequency signal can reach $100 \mathrm{~nm}$, being strongly depends on the contrast of material properties.

Vanadium dioxide is a technologically important material with numerous potential applications in ultrafast modulators, switchable polarizers, beam-steering, thermochromic and others devices. In bulk, this strongly-correlated-electron oxide undergoes a metal-insulator transition at $T_{c}=68{ }^{\circ} \mathrm{C}$ with about five orders of magnitude change in electrical conductivity. The transition is accompanied by a tetragonal-to-monoclinic structural transformation. Due to reduction of the lattice symmetry at the phase transition, the material becomes twinned (divided into orientational domains). In strained quasi-2D nanoplatelets, twins can form quasiperiodic structures as a consequence of selforganization of metal and semiconducting domains [3] during the phase transition on cooling from the growth temperature. Such twins reveal themselves in well-ordered quasiperiodic structures of ridges and furrows in topographic AFM images as visible in Fig. 2(a), with topographic height and lows corresponding to domain walls. Using temperature-dependent SMM imaging, we demonstrate formation of conductive channels at twin boundaries of these structures at temperatures well below bulk transition.

Figure 2(b) shows appearance of metallic domains along ridges at the beginning of the phase transition of heating. At the scan line indicated by the arrow in Fig. 2(c), the temperature was increased by $2{ }^{\circ} \mathrm{C}$, and as is seen, a number of new metallic domains appeared strictly at the ridges of the topographic structure. This mesoscopic-scale phase transition is partially induced by localized strain fields in domain walls due to relatively large clapping angle (obliqueness) of this type of 
twins. The effect can be used to enable new electronic devices though strain engineering of conductive and semiconductive regions. SMM turned to be of particular value in these experiments since it does not require fabrication of contacts, which allows, among other things, preservation of the domain structure in the nanoplatelets.

\section{References}

[1] S. M. Anlage et al., in Scanning Probe Microscopy: Electrical and Electromechanical Phenomena at the Nanoscale, edited by S. Kalinin, and A. Gruverman, Springer Scientific, New York, 2007.

[2] K. Lai et al., Nano Lett. 9, (2009) 1265.

[3] J. Wu et al., Nano Lett. 6, (2006) 2313.

[4] Research at ORNL's Center for Nanophase Materials Sciences was sponsored by the Scientific User Facilities Division, Office of Basic Energy Sciences, U.S. Department of Energy. The research at SIUC was supported through NSF ECCS-0925837 and SISGR-DOE ERKCM67.

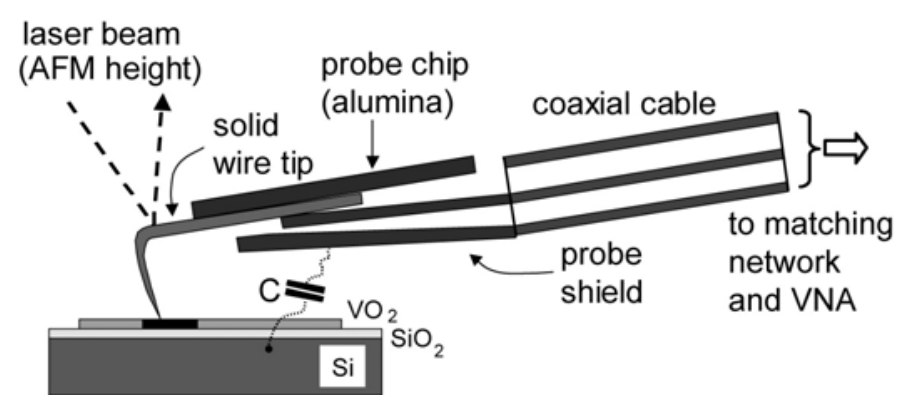

Fig. 1. Schematic of the SMM probe.
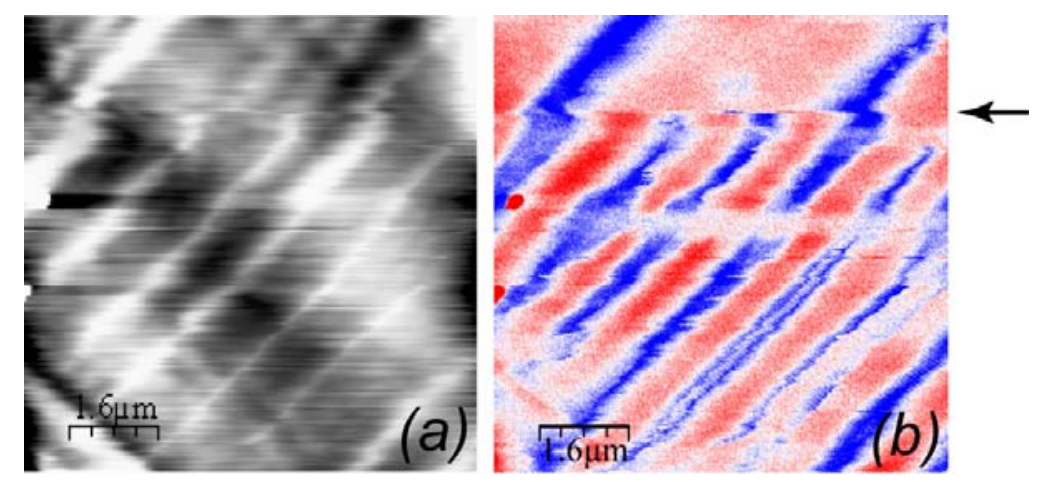

Fig. 2. (a) Topographic AFM image of surface over a quasiperiodic twin structure in a $\mathrm{VO}_{2}$ nanoplatelet and (b) SMM image taken simultaneously with the image in (a). The scan direction is from top to bottom. At the scan line marked by the arrow, the temperature was increased by $2{ }^{\circ} \mathrm{C}$. White in (a) corresponds to larger height; blue (black) in (b) corresponds to larger conductivity. The scale bar is $1.6 \mu \mathrm{m}$. SMM imaging frequency was $1.78 \mathrm{GHz}$. 